\title{
First-principles calculations of the atomic and electronic structure of $F$ centers in the bulk and on the (001) surface of $\mathrm{SrTiO}_{3}$
}

\author{
J. Carrasco, ${ }^{1}$ F. Illas, ${ }^{1, *}$ N. Lopez, ${ }^{1}$ E. A. Kotomin,${ }^{2,3}$ Yu. F. Zhukovskii, ${ }^{2}$ R. A. Evarestov, ${ }^{3,4}$ Yu. A. Mastrikov, ${ }^{3}$ \\ S. Piskunov, ${ }^{2}$ and J. Maier ${ }^{3}$ \\ ${ }^{1}$ Departament de Química Física i Centre especial de Recerca en Química Teòrica, \\ Universitat de Barcelona i Parc Científic de Barcelona, C/Martí i Franquès 1, E-08028 Barcelona, Spain \\ ${ }^{2}$ Institute for Solid State Physics, University of Latvia, Kengaraga 8, LV-1063 Riga, Latvia \\ ${ }^{3}$ Max-Planck-Institut für Festkörperforschung, Heisenbergstrasse 1, D-70569 Stuttgart, Germany \\ ${ }^{4}$ Department of Quantum Chemistry, St. Petersburg State University, Stary Peterhof, 198504 St. Petersburg 198904, Russia
}

(Received 10 June 2005; revised manuscript received 4 November 2005; published 10 February 2006)

\begin{abstract}
The atomic and electronic structure, formation energy, and the energy barriers for migration have been calculated for the neutral $\mathrm{O}$ vacancy point defect $\left(F\right.$ center) in cubic $\mathrm{SrTiO}_{3}$ employing various implementations of density functional theory (DFT). Both bulk and $\mathrm{TiO}_{2}$-terminated (001) surface $F$ centers have been considered. Supercells of different shapes containing up to 320 atoms have been employed. The limit of an isolated single oxygen vacancy in the bulk corresponds to a 270 -atom supercell, in contrast to commonly used supercells containing $\sim 40-80$ atoms. Calculations carried out with the hybrid B3PW functional show that the $F$ center level approaches the conduction band bottom to within $\sim 0.5 \mathrm{eV}$, as the supercell size increases up to 320 atoms. The analysis of the electronic density maps indicates, however, that this remains a small-radius center with the two electrons left by the missing $\mathrm{O}$ ion being redistributed mainly between the vacancy and the $3 d\left(z^{2}\right)$ atomic orbitals of the two nearest Ti ions. As for the dynamical properties, the calculated migration energy barrier in the low oxygen depletion regime is predicted to be $0.4 \mathrm{eV}$. In contrast, the surface $F$ center exhibits a more delocalized character, which leads to significantly reduced ionization and migration energies. Results obtained are compared with available experimental data.
\end{abstract}

DOI: 10.1103/PhysRevB.73.064106

PACS number(s): 31.15.Ar, 61.72.Bb, 61.72.Ji

\section{INTRODUCTION}

Oxides constitute a broad class of materials, which exhibit very attractive possibilities from a technological point of view. $^{1,2}$ Their electrical properties range from insulators to semiconductors and some even exhibit metallic character. These electrical properties together with their (usually) high thermal resistance are fundamental for their use in many applications ranging from protective coatings to constituents in microelectronic devices. Most of the chemical activity and physical properties of oxides can be monitored either by nanostructuring the compounds or through the introduction of intrinsic or extrinsic dopants. ${ }^{3,4}$ This leads to the challenging concept of defect engineering in materials design; this is the control of the nature and concentration of defects in a material in order to tune its properties in a desired manner or to generate a completely unique behavior. The nature of defects in simple ionic oxides has been studied quite extensively, ${ }^{5-10}$ including its effect on the magnetic moment of supported $\mathrm{Ni}$ atoms. ${ }^{11}$ However, much less is known about the nature of point defects in less simple oxides. This is because in the case of covalent and semicovalent oxides$\mathrm{ABO}_{3}$ perovskites among them-their electronic structure exhibits an increased complexity which has hindered a proper characterization of these systems.

Strontium titanate $\left(\mathrm{SrTiO}_{3}\right)$ may serve as a model material in this respect. It exhibits a mixed ionic-covalent chemical bonding since the $\mathrm{Sr}-\mathrm{O}$ bonds are quite ionic while the Ti-O substructure is partly covalently bound. ${ }^{12,13}$ This particular nature of the chemical bonding leads also to a rather unique electronic structure with a band gap of $3.3 \mathrm{eV}$ only. ${ }^{12}$ Hence, as it simultaneously exhibits a strong chemical stability, $\mathrm{SrTiO}_{3}$ continues to attract considerable attention as a model electroceramic material. The investigation of the surface structure and properties of this material is even more difficult. Theoretical calculations carried out for the (001) perfect surfaces terminated either by $\mathrm{TiO}_{2}$ or $\mathrm{SrO}$ planes differ in surface energy by less than $0.1 \mathrm{eV}$ per surface unit cell, indicating that the two terminations are equally stable. ${ }^{14}$ However, due to the partially covalent nature of $\mathrm{TiO}_{2}$-terminated surface, this is more attractive for applications; the reconstructed surface has also been studied. ${ }^{15,16}$ The $F$ centers are formed in strontium perovskite when neutral $\mathrm{O}$ atoms are removed from the lattice sites. ${ }^{7,8}$ These centers strongly perturb the material electronic structure and it becomes even more complex for the O-deficient $\mathrm{SrTiO}_{3}$ material than for the perfect crystal. As electronic and ionic charge carrier concentrations are coupled by defect chemistry and electroneutrality, $\mathrm{O}$ vacancies are not only of direct significance for the ionic mobility (and hence by, e.g., degradation kinetics), but also indirectly for the electronic conductivity. Consequently, considerable experimental efforts were focused on studying and understanding the properties of defective $\mathrm{SrTiO}_{3}$, including the ionic transport ${ }^{17}$ and structural properties. ${ }^{12,18}$ Moreover, oxygen vacancies have been supposed to play an important role in determining the $\mathrm{SrTiO}_{3}$ structure since they appear to modulate the transition temperature between the tetragonal (antiferro-distorted) and the cubic phase, usually taking place around $100 \mathrm{~K} .{ }^{19}$

Compared to simple ionic oxides such as $\mathrm{MgO}$ or $\mathrm{Al}_{2} \mathrm{O}_{3}$, the more complex electronic structure of $\mathrm{SrTiO}_{3}$ has impor- 
tant consequences with respect to the electronic structure of the corresponding $F$ centers. In ionic oxides, the ground state of the $F$ center involves two electrons well localized in the vacancy giving rise to energy levels in the band gap region with typical optical absorption around 5-6 eV.,20-23 For perovskite oxides such as $\mathrm{SrTiO}_{3}$, the electronic structure of the $F$-type defects is a subject of a long debate. ${ }^{24}$ Several theoretical studies have suggested major and identical contributions from the $3 d$ orbitals of the two $\mathrm{Ti}$ ions on both sides of $\mathrm{O}$ vacancy to the electron density of the $F$ center, ${ }^{13,19,26}$ although others indicate a partial or total localization of the electrons in the vacancy. ${ }^{27}$ The position of the $F$ center level in the optical band gap is also somewhat controversial $^{19,25}$ although indirect experimental data based on conductivity measurements suggest that the neutral $F$ center is a quite shallow defect. ${ }^{29}$ Another aspect, which is of interest to many applications, concerns the mobility of $\mathrm{O}$ vacancies since this seems to be the mechanism responsible for the electrical behavior at low partial pressures. ${ }^{17}$ Experiments performed at temperatures high enough so that $F$ centers are supposed to have released their electrons, suggest a value of $0.86 \mathrm{eV}$ for the migration energy in the bulk ${ }^{17}$ whereas semiempirical shell model calculation for the empty vacancy predicts a smaller although close enough value of $0.76 \mathrm{eV}^{28}$

The theoretical study of isolated single defects is not only nontrivial and time consuming but still remains of primary importance, since in most of experiments defect concentrations are quite low, whereas typical calculations are performed for unrealistically high defect concentrations which do not permit a direct comparison with experiments. Hence, a number of theoretical studies dealing with the atomic and electronic structure of the $F$ center in $\mathrm{SrTiO}_{3}$, based on first principles calculations, mainly relying on density functional theory (DFT) and using either cluster or periodic models, have appeared in the last few years. ${ }^{13,19,25,26,29,30}$ Concerning the surface $F$ centers, only two studies are available in the literature, one based on semiempirical methods ${ }^{31}$ and another using $a b$ initio methods but for small supercells. ${ }^{32}$ No first principles calculations of the activation energy for the $F$ center migration have been reported so far. However, a careful analysis of the literature reveals a number of flaws, which require further attention. First, most of the previous studies are based on the use of supercell sizes of typically 40-80 atoms and, second, most of these studies are based on the generalized gradient approach (GGA) of the DFT. Recently it has been shown that the above mentioned supercell sizes are too small to screen the strong interaction of the periodically repeated $F$ centers. ${ }^{13,19}$ Also, it is well known that the GGA typically predicts underestimated values of the band gap of many oxides. ${ }^{33,34}$ On the other hand, the selfconsistent-field Hartree-Fock (SCF-HF) method correctly predicts the insulating character of these compounds but typically overestimates the band gap $^{35}$ as also shown for $\mathrm{SrTiO}_{3} .{ }^{29}$ This artifact of $\mathrm{HF}$ or the GGA, which is even worse in the case of the broadly used local density approach (LDA), prevents a correct prediction of the positioning of the defect level with respect to the conduction band bottom. However, calculations on the (001) surface of $\mathrm{SrTiO}_{3}$ performed using the $\mathrm{LDA}+U$ method have shown that they might be able to partially correct this effect. ${ }^{32}$ Likewise, the so-called hybrid exchange-correlation functionals, which have the common trend of mixing some Hartree-Fock exchange with either LDA or GGA exchange, ${ }^{36}$ seem to give rather accurate optical ${ }^{37,38}$ and magnetic properties of oxides. $^{39-42}$

The aim of the present paper is to obtain accurate values for the defect formation and migration energies as well as an accurate and converged study of the local geometry around the $F$ center, to determine its electronic structure and, finally, the mobility (migration energy) of the single $F$ centers in the cubic phase of $\mathrm{SrTiO}_{3}$ perovskite for both the bulk and the $\mathrm{TiO}_{2}$-terminated (001) surface. To this end very large supercells are used coupled to two different implementations of the DFT using either plane waves or localized atomic orbitals as basis sets. In Sec. II the computational details are described. The main results are presented in Sec. III with particular emphasis on the dependence of the calculated defect properties, the supercell shape, and size. Finally, the interpretation of existing experimental data and conclusions are presented in Sec. IV.

\section{COMPUTATIONAL DETAILS}

The $\mathrm{SrTiO}_{3} F$ centers were modeled by removing a single $\mathrm{O}$ atom from the regular lattice site (Fig. 1). A periodic defect model has been used with rather large supercells containing between 40 and 320 atoms. A need in such large supercells comes from recent studies, which show a considerable dependence of vacancy formation energy and lattice relaxation on the supercell size, or equivalently on the vacancy concentration. ${ }^{13,19}$ This is also in agreement with a recent study on $\mathrm{Fe}$ impurity in $\mathrm{SrTiO}_{3}$ suggesting that the interaction of periodically repeated defects can be quite considerable even for supercells containing 160 atoms. ${ }^{43}$ The corresponding local geometry has been determined by total energy minimization with respect to the coordinates of atoms lying in spheres of increasing radius surrounding the vacancy or all atoms in the supercell. Since the cubic phase is relevant at room temperature, we will consider only this phase, which is also easier computationally.

Two different sets of periodic DFT calculations have been carried out. The first one employs a plane wave basis with a cutoff in the kinetic energy of $415 \mathrm{eV}$, uses the PAW method to represent the inner cores ${ }^{44,45}$ and the PW91 implementation $^{46}$ of the GGA exchange-correlation functional. A Monkhost-Pack ${ }^{47} k$-mesh was employed for the energy evaluation with density ranging from 0.04 to $0.51 \AA^{-1}$ (for each case the actual $k$-point mesh is described in the tables). The electronic structure was studied for the diamagnetic closed shell state since spin-polarized calculations always converged to the spin unpolarized solution. ${ }^{13}$ This set of calculations was carried out with the VASP code. ${ }^{48}$ Here, bulk O-deficient structures were represented by supercells with the following extensions of the primitive unit cell vectors: $2 \times 2 \times n$ with $n=2-7 ; 3 \times 3 \times n$ with $n=3,4,5 ; n \sqrt{2} \times n \sqrt{2}$ $\times n \sqrt{2}$ with $n=2,3 ; 2 \sqrt{3} \times 2 \sqrt{3} \times 2 \sqrt{3}$, and $4 \times 4 \times 3$. This corresponds to the structures of $\mathrm{SrTiO}_{3-\delta}$ with $\delta$ ranging from $4 \%$ to $0.7 \%$. The minimal distance between periodi- 


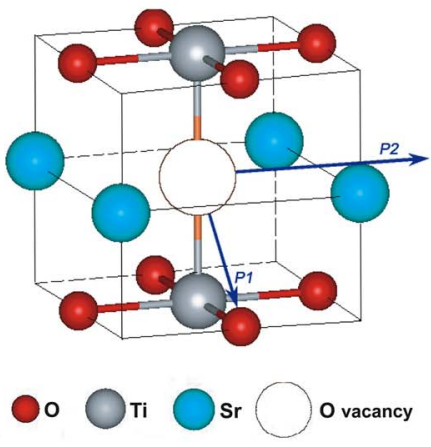

(a)
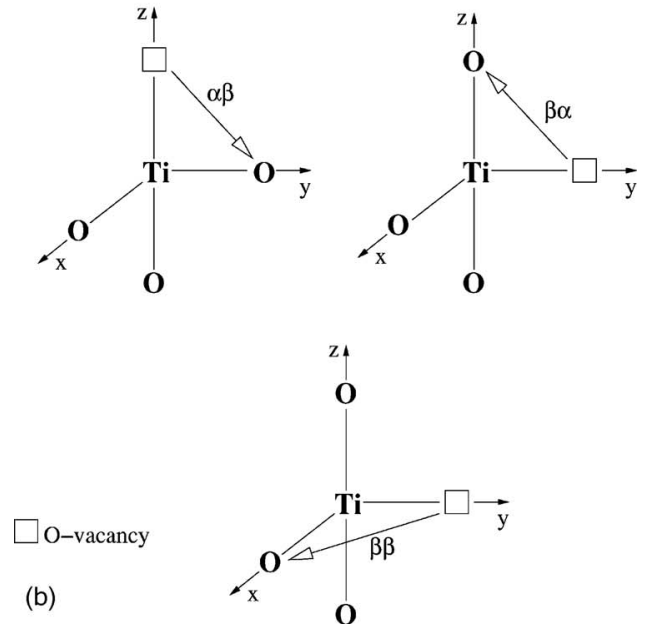

FIG. 1. (Color online) (a) Unit cell of a cubic $\mathrm{SrTiO}_{3}$ with the $F$ center and two probable paths of the (110) and (001) ( $P 1$ and $P 2)$ defect migration. (b) Description of $\alpha$ and $\beta$ O-vacancy defect migration in bulk $\mathrm{SrTiO}_{3}$ for asymmetric cells.

cally repeated defects in the bulk varies from $7.8 \AA$ in the smallest supercell (40 atoms) to $16.6 \AA$ ( 270 atoms) for the largest structure. The main advantage of the VASP computational code is that, upon vacancy creation, it allows one to carry out a very effectively complete lattice relaxation even for large supercells. The scheme presented here is very similar to that employed to identify the structure of isolated vacancies in other oxides. ${ }^{9,49}$ Finally, vacancy migration barriers were calculated as the difference of the total energies for the defect in its equilibrium position and at the saddle point, which is obtained by imposing symmetry constraints as done for other metal oxides. ${ }^{5}$ In both cases, all supercell atoms were relaxed.

The second set of DFT calculations makes use of localized Gaussian-type basis sets and effective core potentials similar to those broadly used in molecular quantum chemistry; the total energy is computed through the hybrid B3PW exchange-correlation functional. ${ }^{50}$ These calculations have been carried out by means of the CRYSTAL- 03 code. ${ }^{51}$ The basis sets for $\mathrm{Sr}$ and $\mathrm{Ti}$ were optimized using small core Hay-Wadt pseudopotentials, ${ }^{52}$ i.e., HWSC-311( $\left.s p\right)-1(d)$ and HWSC-411(sp)-311 $(d)$, respectively, whereas the $\mathrm{O}$ atoms were described by the all-electron basis set $8(s)-411(s p)$ $1(d) .{ }^{53}$ Moreover, a partial reoptimization of the outermost shells of all basis sets mentioned above has been carried out using the PAROPTIMIZE code ${ }^{54}$ interfaced with CRYSTAL-03.
For the study of vacancies in this system, a "ghost" basis set was employed. This means that although the atom is removed, its basis set is left behind; this technique facilitates the correct description of the electron distribution within the vacancy. Ghost basis sets have been successfully employed for the surface $F$ centers on $\mathrm{MgO}$ substrate ${ }^{55}$ and allows for an easy characterization of excited states related to the trapped electrons. ${ }^{20-22}$ To retain cubic symmetry and reduce computational time, this ghost basis set was centered on the core of a missing atom. This does not affect the results obtained. Within this scheme, three types of bulk supercells, all containing a single $F$ center, were considered. These are simple cubic superlattices with the $2 \times 2 \times 2$ and $4 \times 4 \times 4$ expansions of the unit cell (and thus containing 40 and 320 atoms, respectively); fcc supercells with 80 and 270 atoms and, finally, a bcc superlattice with 160 atoms. The distance between periodically repeated defects varies from $7.8 \AA$ for the smallest supercell (40 atoms) to $15.62 \AA$ (320 atoms) in the largest system.

For the simulation of the $\mathrm{SrTiO}_{3}(001)$ surface containing the $F$ center on the $\mathrm{TiO}_{2}$-terminated surface, PW91 and B3PW calculations have been carried out for the 120- and 270-atom supercells, with surface translation vectors of $2 \sqrt{2} \times 2 \sqrt{2}$ and $3 \sqrt{2} \times 3 \sqrt{2}$ extensions, as well as three unit cells (i.e., six atomic planes) along the $z$ direction perpendicular to the surface, respectively, leading to a total defect concentrations ranging from $1.4 \%$ to $0.6 \%$. The minimal distance between the surface defects is $11.1 \AA$ for the 120-atom supercell and $16.6 \AA$ for the 270 -atom one.

\section{RESULTS AND DISCUSSION}

The basic properties of a perfect cubic $\mathrm{SrTiO}_{3}$ bulk and the (001) surface predicted by the DFT methods discussed above have been already reported ${ }^{13,14,53}$ and therefore will only be summarized here. The lattice constant, $a_{0}$, bulk modulus, $B$, as well as elastic constants, $c_{11}, c_{12}$, and $c_{44}$, are close enough to the experimental values in either PW91 or B3PW DFT calculations. However, the electronic structure, and in particular the band gap, is very sensitive to the particular method chosen. The PW91 predicted optical band gap is $2.6 \mathrm{eV}$, or $0.7 \mathrm{eV}$ lower than the experimental one as expected from previous work. ${ }^{38,39}$ A considerable improvement with respect to the experimental band gap is obtained with the $\mathrm{B} 3 \mathrm{PW}$ hybrid functional yielding $3.6 \mathrm{eV}$ vs an experimental value of $3.3 \mathrm{eV} \cdot{ }^{13,53}$ The nature of the chemical bond predicted by the two DFT methods is similar. Effective atomic charges can be obtained by a variety of quite different methods. For instance, the topological charges arising from the Bader analysis ${ }^{56}$ applied to the PW91 density ${ }^{57}$ are $-1.2 e(\mathrm{O}),+2.0 e(\mathrm{Ti})$, and $+1.6 e(\mathrm{Sr})$. The atomic charges arising from the standard Mulliken population analysis applied to the $\mathrm{B} 3 \mathrm{PW}$ density are $-1.42 e(\mathrm{O}),+2.35 e(\mathrm{Ti})$, and $+1.85 e(\mathrm{Sr}){ }^{53}$ Despite a certain numerical difference, these two population analyses are qualitatively similar and both predict a considerable covalency for the Ti-O bond. Recently, an analysis of the effective charges making minimum assumptions and based on the Wannier-type atomic functions has been suggested. ${ }^{58,59}$ This analysis applied to the B3PW 


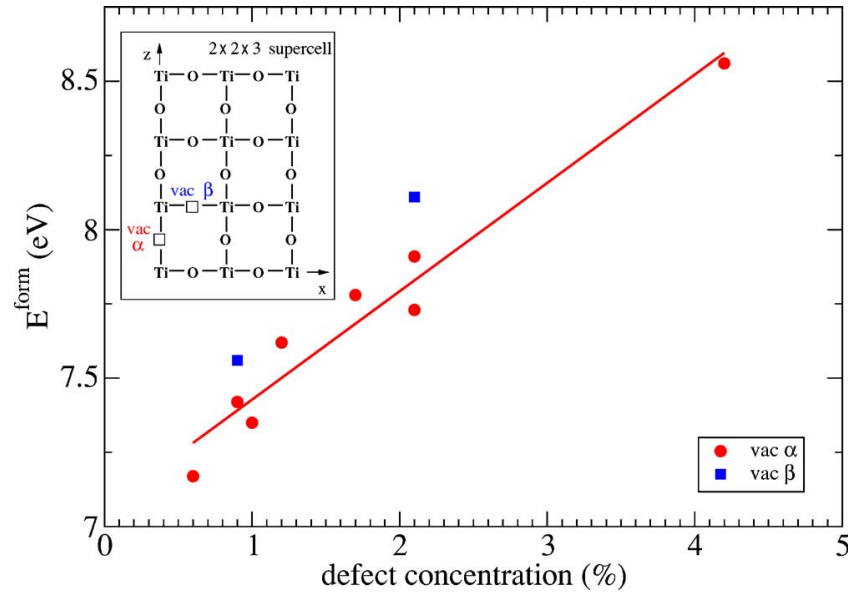

FIG. 2. (Color online) Vacancy formation energy (in eV) versus defect concentration. In all cases only the vacancy formation energies for structures calculated with larger $k$-point meshes are displayed. The corresponding values for very asymmetric supercells are not included. The inset shows the (010) plane for an asymmetric cell, $\alpha$ and $\beta$ vacancies are indicated.

calculations predicts the Sr charge coinciding with the formal charge $(+2 e)$, whereas $\mathrm{Ti}$ and $\mathrm{O}$ ion charges are considerably smaller than the formal ionic charges: $+2.65 e$ and $-1.55 e e^{58}$ respectively. This is also in qualitative agreement with the mixed covalent-ionic character of this compound predicted either by the Bader (PW91) or Muliken (B3PW) charges.

\section{A. Formation energy of neutral oxygen vacancies and lattice relaxation upon vacancy formation}

To estimate the vacancy formation energy of the $\mathrm{O}$ vacancy in the crystal, the following expression is used:

$$
E^{\text {form }}(F)=E(F)+E(O)-E(\text { perfect }),
$$

where $E(O)$ is the spin-polarized energy of an isolated oxygen atom in a ${ }^{3} P$ electronic state, ${ }^{60} E(F)$ and $E$ (perfect) are the energies of the defective and perfect crystal supercells, respectively. Figure 2 clearly demonstrates the sensitivity of the vacancy formation energy to the supercell size. Moreover, this sensitivity is accompanied by a large contribution from the lattice relaxation energy upon vacancy formation. Table I shows that the formation energy is considerably reduced (typically by $1.5 \mathrm{eV}$ ) when the positions of all atoms in the supercells are fully optimized, whereas relaxation of the 14 nearest ions [three nearest Ti-, O-, and Sr-coordination spheres as shown in Fig. 1(a)] is not sufficient. Moreover, the defect formation energy decreases with the increase of the supercell size or, equivalently, by a reduction of the defect concentration (Fig. 2). We consider here the PW91 estimate of $E^{\text {form }}(F)$ since the VASP code allows a complete optimization of the lattice relaxation with a reasonable time and using the parallel version running on 32 processors. The full optimization of the largest supercell took roughly $60 \mathrm{~h}$ in 32 power3 processors of an IBM SP3 machine. Such a geometry optimization procedure for the large supercells is almost impossible with the CRYSTAL code due to enormous computational time needed.

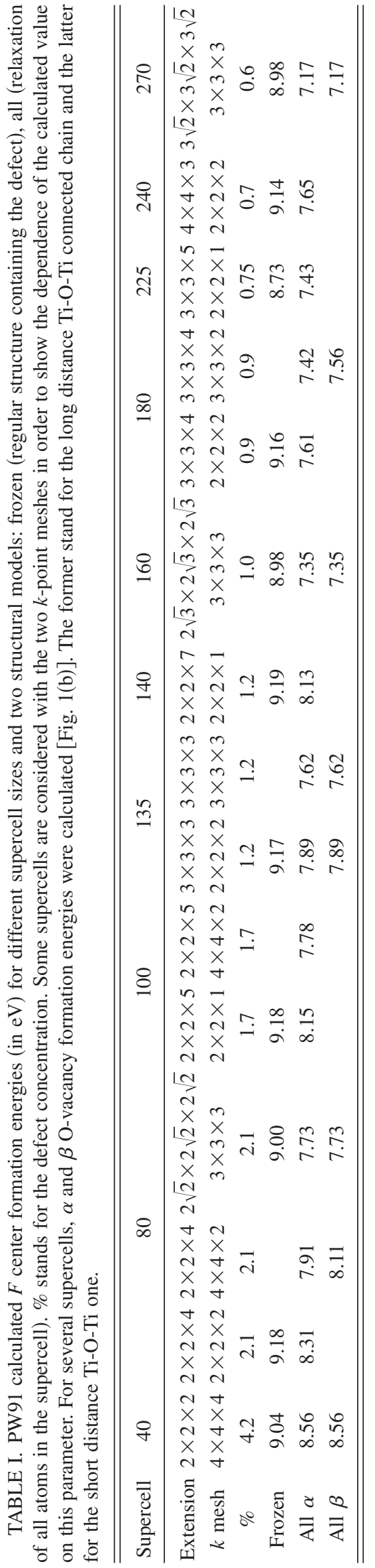


TABLE II. PW91 calculated distances between O vacancy and the nearest Ti, Sr, and O ions (in $\AA$ ) as well as its change (in \%) of the bulk lattice constant $a_{0}$, and the corresponding $\mathrm{O}$ vacancy formation energy for a series of supercells using the $k$-point mesh $2 \times 2 \times 2$. The displacements caused by increasing the number of cells along the $z$, i.e., the (001) direction are marked in bold.

\begin{tabular}{cccccccc}
\hline \hline \multirow{2}{*}{ Atom } & Ideal bulk & $2 \times 2 \times 2$ & $2 \times 2 \times 7$ & $3 \times 3 \times 3$ & $3 \times 3 \times 4$ & $3 \times 3 \times 5$ & $4 \times 4 \times 3$ \\
\hline \multirow{2}{*}{$\mathrm{Ti}$} & 1.961 & $\mathbf{2 . 0 2 6}$ & $\mathbf{2 . 1 3 7}$ & 2.103 & 2.102 & 2.095 & 2.099 \\
& & $\mathbf{1 . 6 7 \%}$ & $\mathbf{4 . 5 1 \%}$ & $3.63 \%$ & $3.60 \%$ & $3.43 \%$ & $3.56 \%$ \\
$\mathrm{O}$ & 2.773 & 2.690 & 2.623 & 2.579 & 2.578 & 2.574 & 2.586 \\
& & $-2.12 \%$ & $-3.85 \%$ & $-4.93 \%$ & $-4.95 \%$ & $-5.07 \%$ & $-4.79 \%$ \\
\multirow{2}{*}{$\mathrm{Sr}$} & 2.773 & 2.841 & 2.865 & 2.864 & 2.873 & 2.873 & 2.876 \\
& & $1.72 \%$ & $2.36 \%$ & $2.32 \%$ & $2.54 \%$ & $2.55 \%$ & $2.64 \%$ \\
\multicolumn{2}{c}{$E^{\text {form }}(F)(\mathrm{eV})$} & 8.94 & 8.13 & 7.89 & 7.61 & 7.43 & 7.65 \\
\hline \hline
\end{tabular}

To obtain the vacancy formation energy at the infinite dilution limit, we have started from the commonly employed $2 \times 2 \times 2$ supercell (40 atoms) corresponding to a vacancy formation energy of $8.56 \mathrm{eV}$ and then enlarged it along the $z$ direction (Ti-O-Ti). The structures being $2 \times 2 \times n$ with $n$ $=2$ up to 7 . This enlargement gives extra stabilization energy of $0.5 \mathrm{eV}$ for the vacancy formation. Second, we have enlarged the supercell on the xy plane normal to the Ti-O axis. The $x, y$ expansions go from $2 \times 2$ to $4 \times 4$ and lead to $\sim 1 \mathrm{eV}$ additional reduction of the formation energy. However, the convergence of the vacancy formation energy to the value of infinite dilution is complex and very large supercells are needed both in the $x, y$, and $z$ directions (Table I). This is in complete agreement with the recent work of Buban et al. ${ }^{19}$ This finding has important consequences since it implies that most of the results reported previously for small supercells containing 40 or 80 atoms are far from being converged to the value at infinite dilution. Probably, even the present results for 320 atom supercells are not completely converged for the isolated single vacancy. Likewise, if one considers that about seven cells are needed along the $z$-direction supercells, more extended supercells along the $x, y$ directions (by $4 \times 4$ or more) are needed to achieve convergence. In addition, careful $k$-point sampling is needed to obtain converged results as can be seen from the comparison between two different point samplings applied to $n \times n \times 4$ and $n \times n \times 5$ supercells (Table I), large $k$-point meshes can reduce the calculated vacancy formation energies by $0.4 \mathrm{eV}$. Extrapolation of the present systematic PW91 values suggests that $7.2 \mathrm{eV}$ is a reasonable estimate for the vacancy formation energy at very low vacancy concentrations. The large increase of the formation energy with respect to the defect concentration seems to be coherent with the experimental indication that above a given vacancy concentration it might be impossible to homogeneously reduce single $\mathrm{SrTiO}_{3}$ crystals and characterize them in terms of standard point defect chemistry. ${ }^{61}$

The strong coupling between the defect formation energy and the local lattice geometry has already been seen earlier for other covalent oxides. ${ }^{5,9}$ For $\mathrm{SrTiO}_{3}$, atomic relaxation extends up to large distances, especially along the $z$ axis (Ti-O-Ti chain). For example, for the $2 \times 2 \times 2$ supercell (Table II), the distance between the neighboring Ti ions and the vacancy center is $0.06 \AA$ larger than that of the Ti-O distance in the perfect structure. However, when the cell is enlarged up to $2 \times 2 \times 7$, this distance is increased up to $0.18 \AA$. Including the second nearest $\mathrm{O}$ neighbors relaxation, the O-O distance, which in the regular structure is $2.77 \AA$, after vacancy formation is reduced so that the $\mathrm{O}-\mathrm{V}_{\mathrm{O}}\left[\mathrm{V}_{\mathrm{O}}\right.$ stands for the missing atom as shown in Fig. 1(a)] distance becomes $2.69 \AA$ for the $2 \times 2 \times 2$ supercell, $2.62 \AA$ for the $2 \times 2 \times 7$ one, and even $2.58 \AA$ for the largest, $4 \times 4 \times 3$ case. As a result, for the largest supercell the change of the Ti$\mathrm{V}_{\mathrm{O}}$ distance is about $3.5 \% a_{0}$ (Table II), comparable to that found for the $\mathrm{Sr}$ ions, but smaller than the $\mathrm{O}$ inward relaxation $\left(4.8 \% a_{0}\right)$.

\section{B. Energy barriers for $\boldsymbol{F}$-center migration}

The energy barriers for the defect migration were estimated by placing the migrating oxygen atom at the saddle point in the plane perpendicular to the line connecting the initial and final $\mathrm{O}$ sites [Fig. 1(a)]. While the coordinates of migrating $\mathrm{O}$ atom were constrained to lie in this symmetry plane, the rest atoms were allowed to relax. Two different paths can be described depending on the planes where the migrating $\mathrm{O}$ atom is moving; these are either $\mathrm{TiO}$ planes (hereafter path 1) or SrO plane (path 2) schematically depicted in Fig. 1(a).

The present calculations carried out for the $2 \times 2 \times 4$, $2 \times 2 \times 5,2 \times 2 \times 6,2 \times 2 \times 7,3 \times 3 \times 3$, and $3 \times 3 \times 4$ supercells predict that the migration in the $\mathrm{SrO}$ planes (path 2) is highly hindered with calculated energy barriers of $\sim 2 \mathrm{eV}$. Consequently, the paths along (001), (100), and (010) directions will not be accessible at room temperatures. For path 1, the calculated barriers at infinite dilution are much smaller (Table III). Unfortunately, migration modeling for the $4 \times 4 \times 4$ and larger supercells could not be accomplished due to the enormous computational cost. For the infinite dilution limit represented by the $3 \times 3 \times 3$ and $3 \times 3 \times 4$ supercells, the barriers are close to $0.4 \mathrm{eV}$, but again, the calculated activation energy depends on both the supercell size and shape. This is because, similarly to the equilibrium state, the atomic relaxations around the interstitial $\mathrm{O}$ ion in the saddle point during the hop towards a vacancy are quite considerable $(0.1-0.3 \AA)$ and extend up to a very long range. However, care must be taken since although all these (110), 
TABLE III. PW91 calculated activation energies $E_{a}$ for the diffusion along (110), (101), and (011) directions, see Fig. 1(b). \% stands for the defect concentration. For totally symmetric supercells, $E_{a}(\alpha \beta)=E_{a}(\beta \alpha)=E_{a}(\beta \beta)$.

\begin{tabular}{ccccc}
\hline \hline Supercell & 40 & 80 & 135 & 180 \\
\hline Extension & $2 \times 2 \times 2$ & $2 \times 2 \times 4$ & $3 \times 3 \times 3$ & $3 \times 3 \times 4$ \\
$k$ mesh & $4 \times 4 \times 4$ & $4 \times 4 \times 2$ & $3 \times 3 \times 3$ & $3 \times 3 \times 2$ \\
$\%$ & 4.2 & 2.1 & 1.2 & 0.9 \\
$E_{a}(\alpha \beta) / E_{a}(\beta \alpha)(\mathrm{eV})$ & 0.61 & $0.55 / 0.35$ & 0.41 & $0.53 / 0.40$ \\
$E_{a}(\beta \beta)(\mathrm{eV})$ & & 0.70 & & 0.42 \\
\hline \hline
\end{tabular}

(011), and (110) different migration directions belonging to path 1 would be equivalent at infinite dilution, they are not at finite concentrations and/or for nonsymmetric unit cells simply because the symmetry is lost. The calculated migration energy barriers are then split into two different subcategories: i.e., (011) and (101) appear to be equivalents in the present supercells but migration along (110) is different [Fig. 1(b)]. This is a clear evidence by the summary of results in Table III: the larger is the vacancy concentration the larger the migration barrier. In particular, for very small unit cells, an energy barrier of $0.7 \mathrm{eV}$ along one of the directions is found.

In order to have an estimate of the influence of the exchange-correlation functional on the calculated migration barriers, the superstructures for the $\mathrm{O}$ vacancy migration were used as external inputs for B3PW single-point frozen geometry CRYSTAL calculations. For the 80 -atom supercell corresponding to a $2 \sqrt{2} \times 2 \sqrt{2} \times 2 \sqrt{2}$ extension, this gives a (110) migration barrier of $0.62 \mathrm{eV}$, which is comparable to the VASP-PW91 value. These concentration effects are related to the possibility of nanoengineering sequences of fully oxidized and oxygen depleted $\mathrm{SrTiO}_{3}$ layers that has been recently reported. ${ }^{18}$ Finally, it is worth pointing out that the present infinite dilution energy barrier is smaller than the one detected experimentally for the migration in the bulk $(0.86 \mathrm{eV}) .{ }^{17}$ However, special care must be taken when comparing to experiment since that was performed at high temperature and under such conditions the $F$ centers already released their electrons to the conduction band. ${ }^{17}$ This is consistent with the semiempirical shell model calculations for the empty oxygen vacancy in a $\mathrm{SrTiO}_{3}$ cubic crystal, which predict the migration barrier of $0.76 \mathrm{eV}$, closer to experiment. $^{28}$

\section{Electronic structure of O-deficient $\mathrm{SrTiO}_{3}$}

The defect-defect interaction in the supercell model is clearly seen when analyzing the calculations carried out with a localized basis set. Therefore, most of the discussion in this section corresponds to the results obtained with the B3PW method. Let us recall that this method predicts a very reasonable estimate of the optical band gap whereas the PW91 method (and in general all GGA methods) predicts a significantly underestimated value of $2.1 \mathrm{eV}$ for this measurable quantity and hence it is very likely that the electronic struc- ture related to the oxygen vacancy will be qualitatively wrong.

The redistribution of the $\mathrm{B} 3 \mathrm{PW}$ electronic density due to the vacancy formation is plotted in Fig. 3 for the 160 and 320 atom supercells. When forming the $\mathrm{O}$ vacancy, the two electrons associated with a removed $\mathrm{O}$ atom are mainly localized on the $3 d\left(z^{2}\right)$ orbitals of the two neighboring titanium ions (well-localized charge redistribution is limited by $\pm 1.5 a_{0}$ in the $z$ direction), thus making the largest contribution to the defect band shown in Fig. 4. This is in agreement with the most of previous $a b$ initio calculations ${ }^{13,25,26}$ but is in contradiction with the recent study of Buban et al. ${ }^{19}$ This latter point will be further discussed in Sec. IV. Table IV and Fig. 4 show that the width (dispersion) of the defect band for commonly used 40-atomic supercells is $0.41 \mathrm{eV}$ and remains still large, $0.23 \mathrm{eV}$, for 135 -atomic supercell corresponding to the $F-F$ distances of $7.8 \AA$ to $11.71 \AA$. This shows that defects are still interacting laterally along the $x, y$ directions and that this interaction has a marked electronic character; it is not only a result of the strong geometry relaxation induced by the presence of the oxygen vacancy.

The energy band corresponding to the $F$ center is split off the conduction band. We calculated its depth relatively to the bottom of the conduction band at the $\Gamma$ point of the Brillouin zone (Fig. 4). In the limit of the isolated single defect, the defect band has to transform into an energy level. The defect ionization energy $\left(\delta \varepsilon_{d b \text {-gap }}^{\Gamma}\right.$ in Fig. 4$)$ is reduced from $1.11 \mathrm{eV}$ (40 atom) down to $0.72 \mathrm{eV}$ (135 atom), respectively. Last, for the 270 and 320 atom supercells, the defect band indeed is degenerated into the energy level, which is separated from the conduction band bottom by $0.49 \mathrm{eV}$. Negligible dispersion of the defect band for 270- and 320-atom supercells (0.02 and $0.03 \mathrm{eV}$, respectively) means that the defect-defect interaction is practically eliminated, thus approaching the desired isolated single $F$ center limit.

The estimated ionization energy of $0.5 \mathrm{eV}$ is in a sharp contrast with the order-of-magnitude larger values observed for the $F$ centers in ionic oxides ${ }^{7}$ but still much larger than the experimental estimate based on conductivity measurements, i.e., $0.03 \mathrm{eV} .{ }^{27}$ The discrepancy with experiments could be due to several reasons: (i) experimental estimates are based on indirect conductivity measurements, (ii) $\mathrm{O}$ vacancies effectively aggregate, (iii) experimentalists estimate in fact the thermal ionization energy of the $F$ center which is typically smaller than difference of one-electron energies in our case, (iv) the band gap in our hybrid linear combination of atomic orbitals (LCAO) calculations is state-of-the-art value but still exceeds the experimental value by $0.3 \mathrm{eV}$ which could be crucial in this case: corrected position of the $F$ center level could be as small as $0.2 \mathrm{eV}$.

A more reliable characterization of the $F$ center and whether it is shallow or deep is provided by the analysis of its wave function localization. As we discussed above, the present calculations for the largest supercell of 320 atoms [Fig. 3(b)] clearly show that the $F$ center density is well localized on the vacancy and the two nearest Ti ions. This is in contrast with an earlier conclusion made in the LDA plane wave calculations for 320 -atom supercells. ${ }^{19}$ The differences arise very likely from the large band gap underestimation by the LDA and its trend to excessively delocalize the electron 
a)

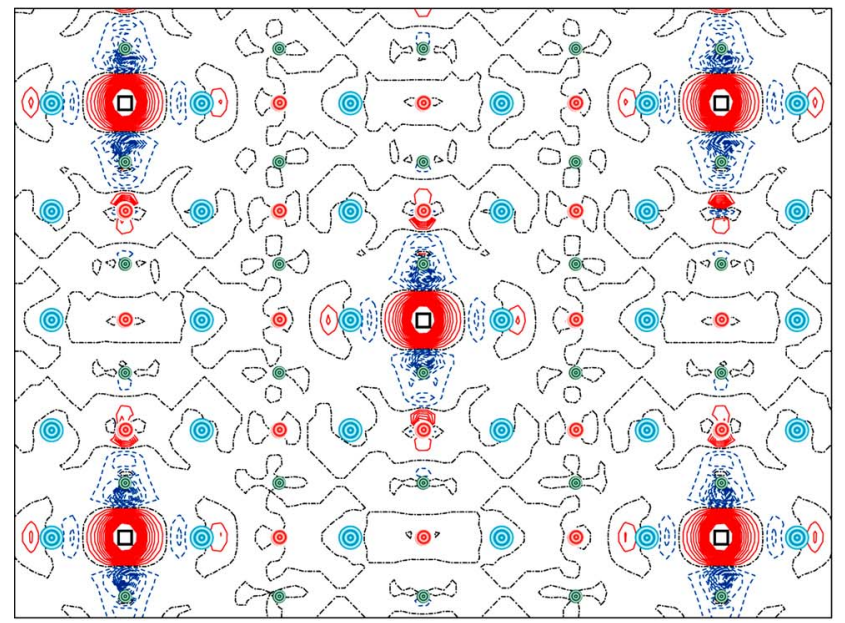

b)

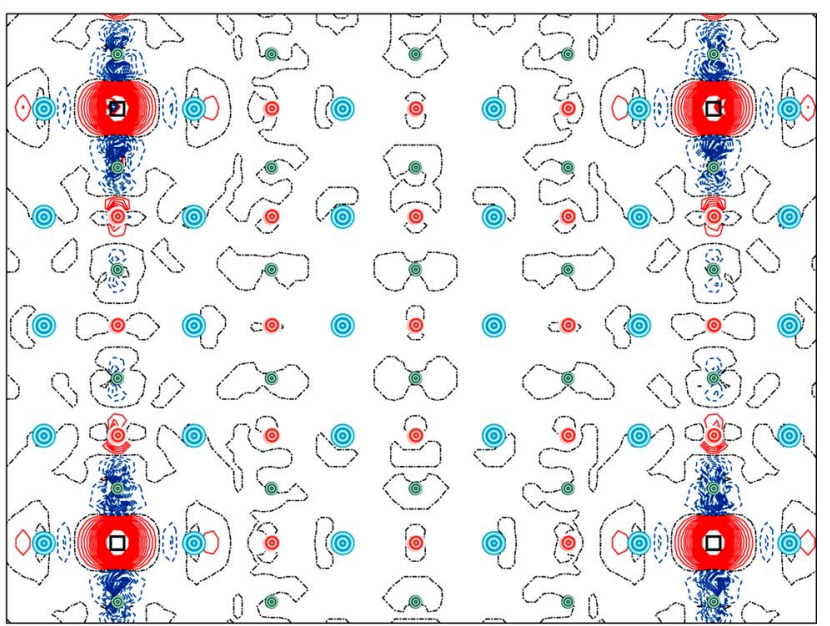

() $\mathrm{Sr}$ (ㅇ $\mathrm{O}$ (2) $\mathrm{Ti} \square \mathrm{O}$ vacancy

FIG. 3. (Color online) The B3PW calculated (110) cross section of the difference electron density maps $\Delta \rho(\mathbf{r})$ for two different supercells containing a single oxygen vacancy: (a) 160-atomic bcc cell; (b) 320-atomic sc cell. $\Delta \rho$ (r) is defined as the total density in the perfect $\mathrm{SrTiO}_{3}$ bulk minus the sum of the electron densities for the isolated oxygen atoms and those of the defective $\mathrm{SrTiO}_{3}$. Dash-dot (black) isolines correspond to the zero level. Dotted (blue) isolines stand for an decrease in $\Delta \rho(\mathbf{r})$ and solid (red) lines for a increase.

density. ${ }^{40,62,63}$ The lattice relaxation using complete optimization of supercell structure can affect the LCAO defect bandwidth and its position with respect to the conduction band bottom. The localization of the electrons on titanium ions is consistent with photoemission studies on the surface of $\mathrm{SrTiO}_{3}$, which indicate the presence of $\mathrm{Ti}^{3+}$ ions when surface defects are created under $\mathrm{Ar}^{+}$ion bombardment. ${ }^{64}$

Next, it is worth analyzing also the PW91 electronic structure even, as mentioned above, the systematic underestimate of the band gap implies several difficulties in the description of the electronic structure of defective $\mathrm{SrTiO}_{3}$. Nevertheless, to get some insight in the problem, we have analyzed the total density of states (DOS) and the atomprojected DOS of different O-deficient structures for the perfect structure and for two different O-vacancy concentrations, namely $4.0 \%$ and $0.7 \%$; results are displayed in Fig. 5. The projected DOS shows that the contribution of the two Ti atoms, which are the first neighbors to the vacancy, to the

a)

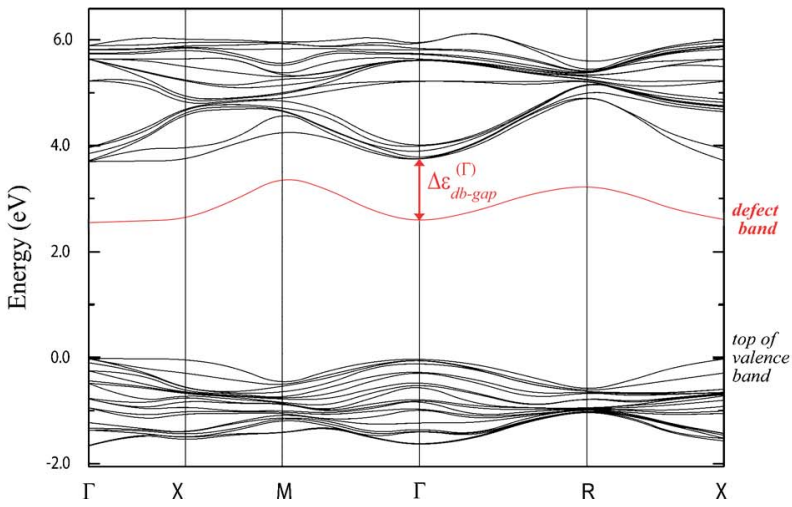

lower part of the conduction band depends strongly on the overall vacancy concentration. For a high O-vacancy concentration, the bottom conduction band (CB) has a large contribution from these two $\mathrm{Ti}$ atoms showing a rather strong interaction between them, this is not the case for the diluted system. For the former case, a wideband is seen with contributions mainly from the two $\mathrm{Ti}$ atoms nearest to the oxygen vacancy. This is a very strong band close to the bottom of the conduction band. As can be seen from Table II, the high vacancy concentration in the $2 \times 2 \times 2$ supercell induces a rather short Ti-Ti distance as compared to the $3 \times 3 \times 5$ supercell. In the latter case, the Ti-Ti distance is elongated by $0.08 \AA$ thus explaining the weaker $F-F$ interaction found in the projected DOS. For the lower vacancy concentration, the levels produced by the $\mathrm{Ti}$ atoms in the neighborhood of the vacancy appear already inside the conduction band. Both behaviors have been described before (isolated impurity state and states inside the band gap). As stated above, one cannot

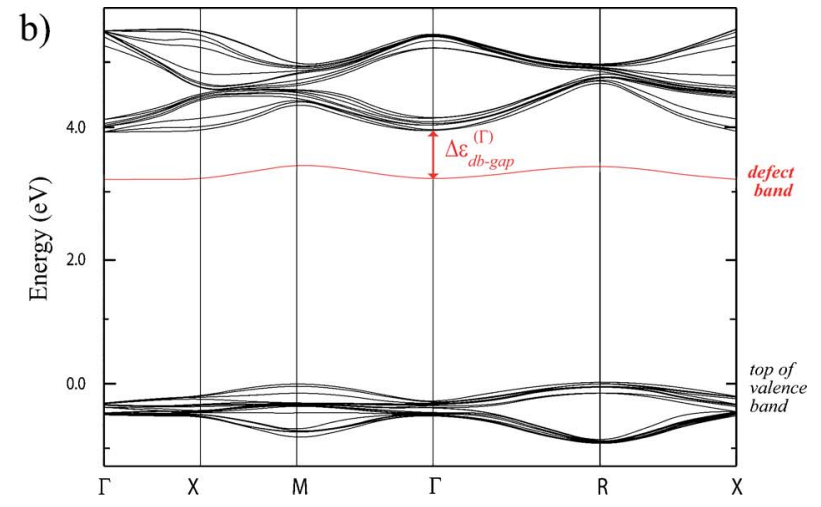

FIG. 4. (Color online) The B3PW band structure of a single $F$ center per sc supercell containing (a) 40 atoms and (b) 135 atoms. 
TABLE IV. Dependence of the $F$ center energy level position with respect to the conduction band $\left(\Delta \varepsilon_{d b \text {-gap }}^{\Gamma}\right.$ in Fig. 4), its dispersion $\left(\delta \varepsilon_{d b \text {-gap }}\right)$, and the distance between nearest $F$ centers $\left(d_{F-F}\right)$ as a function of the supercell size used in the CRYSTAL-B3PW calculations with $2 \times 2 \times 2 \mathrm{k}$ mesh. The defect level position is calculated at the $\Gamma$ point of the first Brillouin zone.

\begin{tabular}{cccccc}
\hline \hline Supercell & Extension & Lattice type & $\begin{array}{c}d_{F-F} \\
(\AA)\end{array}$ & $\begin{array}{c}\Delta \varepsilon_{d b \text {-gap }}^{\Gamma} \\
(\mathrm{eV})\end{array}$ & $\begin{array}{c}\delta \varepsilon_{d b \text {-gap }} \\
(\mathrm{eV})\end{array}$ \\
\hline 40 & $2 \times 2 \times 2$ & $\mathrm{sc}$ & 7.805 & 1.108 & 0.41 \\
80 & $2 \sqrt{2} \times 2 \sqrt{2} \times 2 \sqrt{2}$ & $\mathrm{fcc}$ & 11.04 & 0.692 & 0.15 \\
135 & $3 \times 3 \times 3$ & $\mathrm{sc}$ & 11.71 & 0.721 & 0.23 \\
160 & $2 \sqrt{3} \times 2 \sqrt{3} \times 2 \sqrt{3}$ & $\mathrm{bcc}$ & 13.52 & 0.572 & 0.09 \\
270 & $3 \sqrt{2} \times 3 \sqrt{2} \times 3 \sqrt{2}$ & $\mathrm{fcc}$ & 16.56 & 0.493 & 0.02 \\
320 & $4 \times 4 \times 4$ & $\mathrm{sc}$ & 15.61 & 0.487 & 0.03 \\
\hline \hline
\end{tabular}

enter very deep into this discussion because of the limitations of the PW91 functional. In any case, the displacement of the band with the concentration seems an important, physically meaningful effect.

\section{The $F$ centers on the $(001) \mathrm{TiO}_{2}$-terminated surface}

The atomic and electronic structure of surface $F$ centers is practically unknown. We mentioned already two theoretical papers which dealt with the analysis of the $F$ centers on the $\mathrm{TiO}_{2}$-terminated $\mathrm{SrTiO}_{3}(001)$ surface: one of them was based on semiempirical Intermediate Neglect of Differential Overlap (INDO) calculations ${ }^{31}$ and another one on GGA and LDA $+U$ slab calculations. ${ }^{32}$ In both papers, the structure of the $F$ centers was studied for very high defect concentrations. It has been shown in the previous sections that the results for low defect concentrations in bulk might be significantly different from those corresponding to the infinite di- lution limit. Moreover, it is very likely that in the calculations $^{30,31}$ both lattice relaxation and vacancy formation energies are not converged to the infinite dilution limit and no surface defect migration energies were presented. In the present work, we have studied the surface $F$ centers on the $\mathrm{TiO}_{2}$-terminated unreconstructed (001) surface. This choice is motivated by its abundance at normal air pressures and its common nature for all titanium perovskites. A stoichiometric slab containing six atomic $\mathrm{TiO}_{2}$ and $\mathrm{SrO}$ planes has been chosen as a material model.

The formation energy for relaxed (unrelaxed) surface oxygen vacancies was found to be $6.22 \mathrm{eV}(8.86 \mathrm{eV})$ and $5.94 \mathrm{eV}(8.81 \mathrm{eV})$ for 120-atom and 270-atom supercells, respectively. Again, one must be aware of the fact that the present values might not be completely converged to the infinite dilution limit. This surface vacancy formation energy could be compared to the $7.73 \mathrm{eV}$ and $7.17 \mathrm{eV}$ formation energies for the relaxed $F$ centers in the bulk, calculated for the bulk supercells $n \sqrt{2} \times n \sqrt{2} \times n \sqrt{2}, n=2,3$, with similar interdefect distances along the $z$ axis. The conclusion could be drawn that the defect formation energy on the $\mathrm{TiO}_{2}$-terminated surface is considerably smaller than in the bulk; it is roughly reduced by $\sim 1.5 \mathrm{eV}$ or $\sim 20-25 \%$. This is similar to what has been obtained for other oxides and it is due to the reduced coordination at the surface. ${ }^{9,65}$ The relaxation of the atoms nearest to the vacancy and in the Ti-O-Ti surface chain is reported in Table $\mathrm{V}$. The Ti and $\mathrm{O}$ nearest neighbors of the vacancy are displaced by $\sim 7 \% a_{0}$ and $4 \% a_{0}$, respectively. The former displacements exceed by a factor of two those in the bulk and result from the half coordination sphere left when the surface is formed. Unlike the bulk, where atoms move towards and outwards $\mathrm{O}$ vacancy, on the surface direction of atomic displacements is more complicated (Table V). A well-pronounced strong anisotropy in the atomic displacements along the $\mathrm{Ti}-\mathrm{V}_{\mathrm{O}}$-Ti axis is clearly observed. Atomic displacements in the smaller supercell show a nonmonotonic

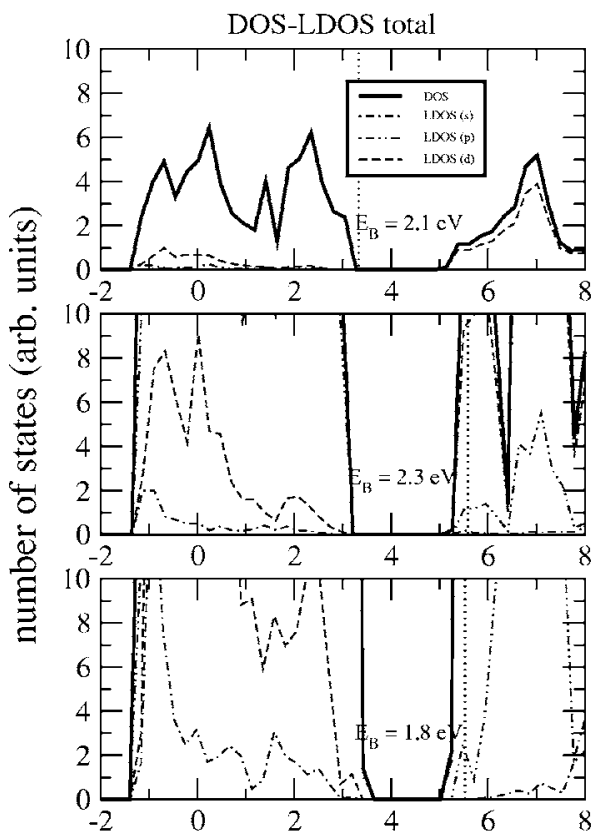

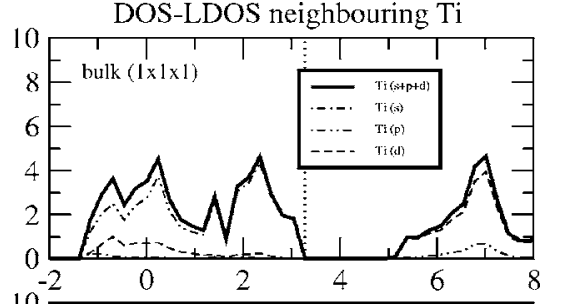
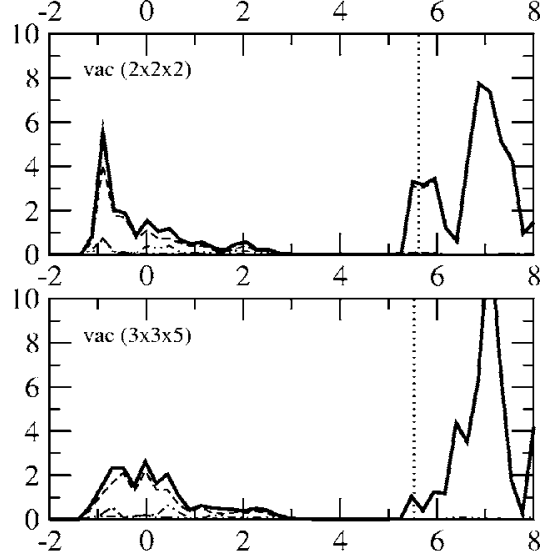

$\mathrm{E}(\mathrm{eV})$
FIG. 5. The PW91 total density of states and projected density of states for the $F$ centers in two different unit cells. Dotted lines indicate the last occupied state. 


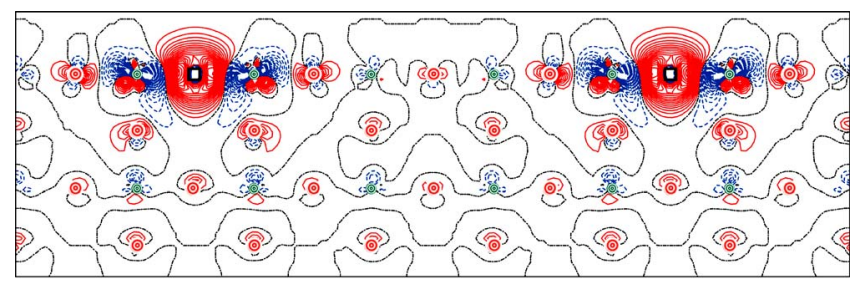

(ㅇ () Ti $\square$ O vacancy

FIG. 6. (Color online) The B3PW (100) cross section of the difference electron density map $\Delta \rho(\mathbf{r})$ for the 120-atomic slab supercell $(2 \sqrt{2} \times 2 \sqrt{2} \times 3$ extension $)$ with a surface oxygen vacancy. See caption of Fig. 3 for an explanation.

decay with the distance from the vacancy (e.g., surface oxygen atom separated from a vacancy by $2 a_{0}$ ), which is caused by the interference effects on the border of the nearest supercells and demonstrates that this supercell is not big enough to avoid defect-defect interactions.

To estimate the barrier for the $F$ center migration on the surface, we followed the same procedure as for the bulk (Sec. III B). The calculated activation energy for defect migration for the two supercells of 120 and 270 atoms is 0.19 and $0.11 \mathrm{eV}$, respectively. This demonstrates that (i) an increase of the distance between defects reduces the migration energy (due to reduced repulsion energy between periodically distributed defects), and (ii) compared to the bulk migration, defect migration energy on the perovskite surface is largely reduced. This is in a line with the recent calculations of the $F$ center migration on the $\mathrm{MgO}$ surface ${ }^{65}$ where the activation energy was obtained also considerably smaller than in the bulk. High vacancy mobility on the $\mathrm{TiO}_{2}$ surface at room temperature could partially explain the difficulties in the scanning tunneling microscopy (STM) study of the $\mathrm{SrTiO}_{3}$ surface. ${ }^{15}$ In addition, high vacancy mobility makes the surface reconstruction easier seen in the experiments.

With respect to the electronic structure of the surface, Fig. 6 presents the rearrangement of the electronic density upon vacancy formation at the $\mathrm{TiO}_{2}$-terminated (001) surface. The difference electronic density map for the surface $F$ center on the $2 \sqrt{2} \times 2 \sqrt{2} \times 3$ slab shows that the electronic density around it is more delocalized than that corresponding to the bulk $F$ center. The calculated Bader effective charges ${ }^{56}$ of the two nearest Ti atoms give an electronic density increase of $0.4 e$ per atom, whereas the rest of the missing $\mathrm{O}$ charge is spread in the vicinity of the vacancy (Fig. 6). Last, Fig. 7 shows that the defect ionization energy $\Delta \varepsilon_{d b \text {-gap }}^{\Gamma}$ of the surface $F$ center is almost twice as small $(0.25 \mathrm{eV})$ than that in the bulk $(0.49 \mathrm{eV})$. Its dispersion is still not negligible $(0.14 \mathrm{eV})$, being comparable with that for the bulk $2 \sqrt{2}$ $\times 2 \sqrt{2} \times 2 \sqrt{2}$ supercell $(0.15 \mathrm{eV})$.

\section{CONCLUSIONS}

The main conclusions of the present calculations regarding the stability, migration, and the electronic structure of bulk and surface $F$ centers of $\mathrm{SrTiO}_{3}$ can be summarized as follows.

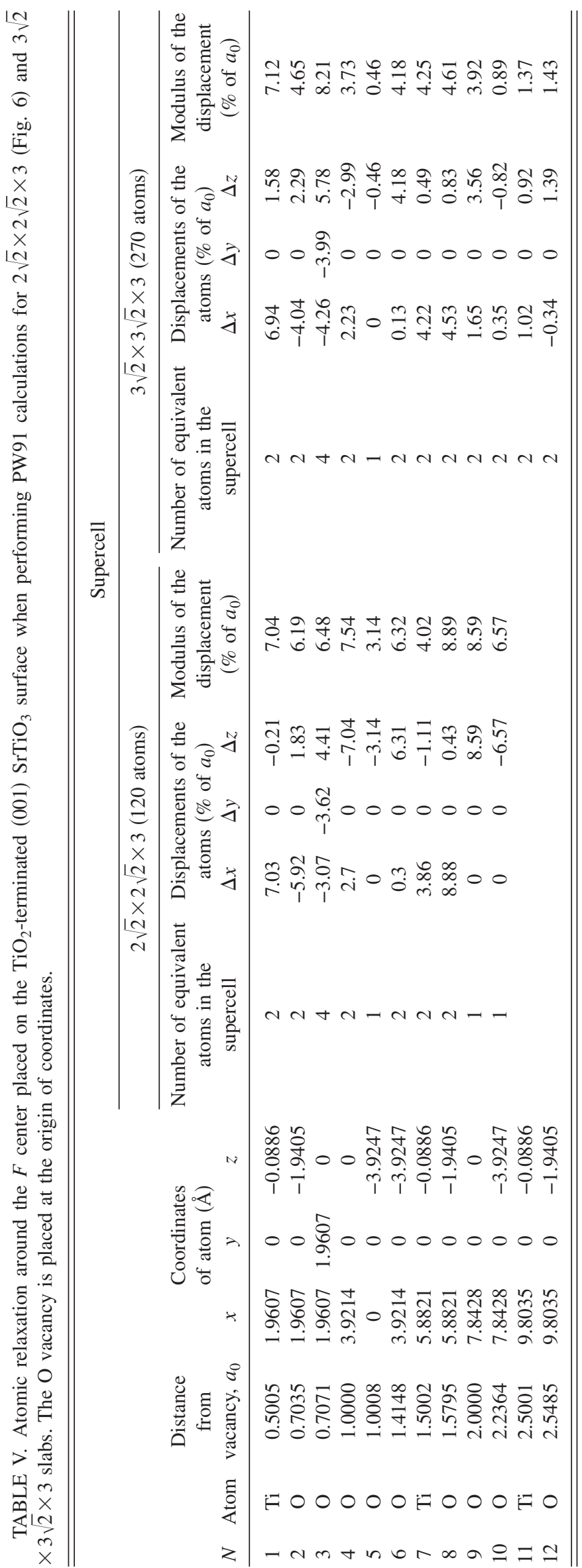




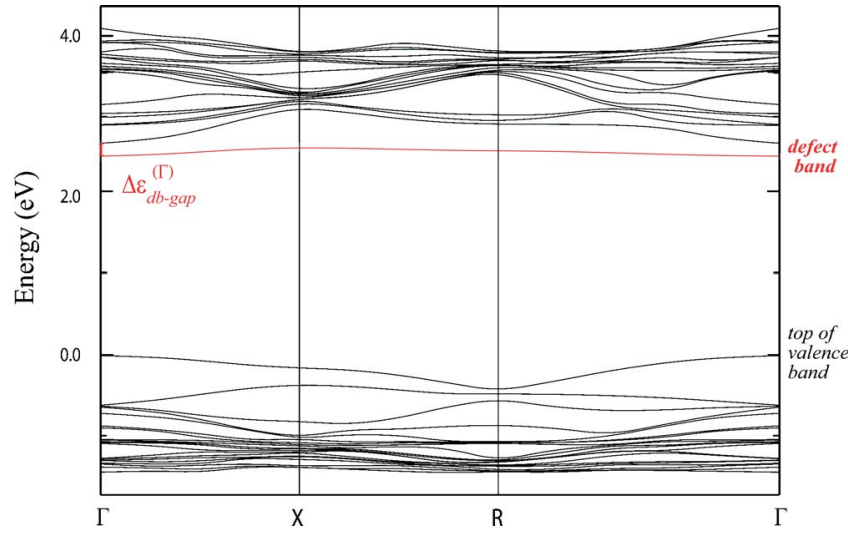

FIG. 7. (Color online) The B3PW band structure of the (001) surface of a cubic $\mathrm{SrTiO}_{3}$ crystal containing the $F$ center in 120atomic supercell. See caption of Fig. 4 for an explanation.

(1) Large bulk supercells containing at least 270 atoms are required to obtain converged results for the lattice relaxation, vacancy formation energies and the electronic properties of oxygen vacancies in $\mathrm{SrTiO}_{3}$. This suggests revising the results obtained earlier using much smaller supercells. Note that the use of noncubic fcc supercells allows one to enlarge the distance between the nearest defects as compared to cubic sc supercells (cf. 4.21 $a_{0}$ and $4.00 a_{0}$ for 270- and 320atom supercells, respectively). This is most important for defects with anisotropic lattice relaxation, like the $F$ centers studied in the present work, where interdefect distance along the $z$ axis in the Ti-O-Ti direction should be as large as possible. However, such supercells are less useful for evaluation of the vacancy migration energies, since the initial and final atomic positions lie in the two chains that are perpendicular to each other [Fig. 1(b)]. In any case, the calculated vacancy formation energy is highly dependent on the defect concentration. On the surface, the vacancy formation energy is by $\sim 1.5 \mathrm{eV}$ smaller due to the reduced atomic coordination.

(2) The migration energy also strongly depends on the supercell size and shape, due to variation in lattice distortion in the ground and especially in the saddle point. An approximation to the value extrapolated to the single defect limit is $0.4 \mathrm{eV}$, which is smaller than the experimental estimate of $0.86 \mathrm{eV} .{ }^{17}$ This discrepancy could arise due to the fact that experimental value refers to the empty $\left(F^{2+}\right)$ charged vacancy without electrons. Resolving this problem would require to carry out first principles calculations for the charged vacancy migration. However, this is not a simple task because of the long-range Coulomb interaction between the charged defects. Last, the migration energy for the surface $F$ center is predicted to be as small as $0.1-0.2 \mathrm{eV}$. This means that defects play an important role in surface-related processes.

(3) The present B3PW hybrid calculations show formation of the $F$ center defect band below the conduction band bottom, which is mainly composed of $3 d\left(z^{2}\right)$ orbitals of the two nearest $\mathrm{Ti}$ ions. These orbitals are considerably mixed with the $s$-type orbitals centered at the vacancy. This is also confirmed by the electron charge redistributions drawn for the two different defect concentrations shown in Fig. 3. Both Ti ions give equal contributions to the $F$ center density. To get more accurate position of the defect level with respect to the conduction band bottom, large-scale structure optimization of the 320-atom supercells within the LCAO implementation of this functional would be necessary. This is beyond our current computational facilities.

(4) The single $F$ center is a small-radius defect. This is qualitatively similar to that found for the same defect in other perovskites such as $\mathrm{BaTiO}_{3}, \mathrm{PbTiO}_{3}$, and $\mathrm{KNbO}_{3}$ (Refs. 25 and 66) although those have been studied for much higher defect concentrations. The surface $F$ center is predicted to be more delocalized than that in bulk. This is in agreement with previous findings for ionic oxides such as $\mathrm{MgO}$ (Ref. 7) or $\mathrm{Al}_{2} \mathrm{O}_{3}$ (Ref. 9) and results from the reduced Madelung potential and atomic coordination at the surface.

\section{ACKNOWLEDGMENTS}

Financial support from the Spanish DGICYT, Generalitat de Catalunya (GC), German-Israeli Foundation (GIF) and HPC-Europa (Project Nos. BQU2002-04029-CO2-01, 2001SGR-00043, and G-703.41.10) is acknowledged. J.C. and N.L. thank the Spanish Ministry of Education for financial support through the Ramon y Cajal and predoctoral programs, respectively. Supercomputer time has been provided by the CEPBA-IBM-Research-Institute and HPC-Europa. F.I. and N.L. acknowledge additional funding from the Distinció de la GC per a la Promoció de la Recerca Universitària. R.E. acknowledges support from the A. von Humboldt Foundation. Y.M. thanks the group members of Quantum Chemistry of Materials at the University of Barcelona for their warm hospitality and training during his visit there. The authors kindly thank O. F. Schirmer, R. de Souza, and E. Heifets for valuable discussions.

\footnotetext{
*Electronic address: francesc.illas@ub.edu

${ }^{1}$ V. E. Henrich and P. A. Cox, The Surface Science of Metal Oxides (Cambridge University Press, Cambridge, 1994).

${ }^{2}$ The Chemical Physics of Solid Surfaces-Oxide Surfaces, edited by P. Woodruff, Vol. 9 (Elsevier, Amsterdam, 2001).

${ }^{3}$ A. Corma, P. Atienzar, H. Garcia, and J.-Y. Chane-Ching, Nat. Mater. 3, 304 (2004).
}

${ }^{4}$ R. Schaub, P. Thostrup, N. Lopez, E. Lægsgaard, I. Stensgaard, J. K. Nørskov, and F. Besenbacher, Phys. Rev. Lett. 87, 266104 (2001).

${ }^{5}$ A. M. Ferrari and G. Pacchioni, J. Phys. Chem. 99, 17010 (1995).

${ }^{6}$ J. Carrasco, N. Lopez, and F. Illas, Phys. Rev. Lett. 93, 225502 (2004).

${ }^{7}$ P. Mori-Sanchez, J. M. Recio, B. Silvi, C. Sousa, A. Martin Pen- 
das, V. Luaña, and F. Illas, Phys. Rev. B 66, 075103 (2002).

${ }^{8}$ E. A. Kotomin and A. I. Popov, Nucl. Instrum. Methods Phys. Res. B 141, 1 (1998).

${ }^{9}$ J. Carrasco, J. R. B. Gomes, and F. Illas, Phys. Rev. B 69, 064116 (2004).

${ }^{10}$ R. A. Evarestov, Phys. Status Solidi A 202, 235 (2005).

${ }^{11}$ N. Lopez, J. C. Paniagua, and F. Illas, J. Chem. Phys. 117, 9445 (2002).

${ }^{12}$ K. van Benthem, C. Elsaesser, and R. H. French, J. Appl. Phys. 90, 6156 (2001).

${ }^{13}$ J. Carrasco, F. Illas, N. Lopez, E. A. Kotomin, Yu. F. Zhukovskii, S. Piskunov, J. Maier, and K. Hermansson, Phys. Status Solidi C 2, 153 (2005).

${ }^{14}$ S. Piskunov, E. A. Kotomin, E. Heifets, J. Maier, R. I. Eglitis, and G. Borstel, Surf. Sci. 575, 75 (2005).

${ }^{15}$ M. R. Castell, Surf. Sci. 516, 33 (2002).

${ }^{16}$ N. Erdman, K. R. Poeppelmeier, M. Asta, O. Warschkow, D. E. Ellis, and L. D. Marks, Nature (London) 419, 55 (2002); Surf. Sci. 573, 446 (2004).

${ }^{17}$ I. Denk, W. Munch, and J. Maier, J. Am. Ceram. Soc. 78, 3265 (1995).

${ }^{18}$ D. A. Muller, N. Nakagawa, A. Ohtomo, J. L. Grazul, and H. Y. Hwang, Nature (London) 430, 657 (2004).

${ }^{19}$ J. P. Buban, H. Iddir, and S. Ogut, Phys. Rev. B 69, 180102(R) (2004).

${ }^{20}$ F. Illas and G. Pacchioni, J. Chem. Phys. 108, 7835 (1998).

${ }^{21}$ C. Sousa, G. Pacchioni, and F. Illas, Surf. Sci. 429, 217 (1999).

${ }^{22}$ C. Sousa and F. Illas, J. Chem. Phys. 115, 1435 (2001).

${ }^{23}$ J. Carrasco, N. Lopez, C. Sousa, and F. Illas, Phys. Rev. B 72, 054109 (2005).

${ }^{24}$ S. Lenjer, O. F. Schirmer, H. Hesse, and Th. W. Kool, Phys. Rev. B 70, 157102 (2004).

${ }^{25}$ H.-J. Donnenberg, Atomic Simulations of Electro-Optical and Magneto-Optical Materials, Springer Tracts in Modern Physics, Vol. 151 (Springer, Berlin, 1999).

${ }^{26}$ T. Tanaka, K. Matsunaga, Y. Ikuhara, and T. Yamamoto, Phys. Rev. B 68, 205213 (2003).

${ }^{27}$ R. Moos and K. H. Härdtl, J. Am. Ceram. Soc. 80, 2549 (1997).

${ }^{28}$ J. Crawford and P. W. M. Jacobs, J. Solid State Chem. 144, 423 (1999).

${ }^{29}$ D. Ricci, G. Bano, G. Pacchioni, and F. Illas, Phys. Rev. B 68, 224105 (2003).

${ }^{30}$ W. Luo, W. Duan, S. G. Louie, and M. L. Cohen, Phys. Rev. B 70, 214109 (2004).

${ }^{31}$ A. Stashans, F. Erazo, J. Ortiz, and P. Valverde, Philos. Mag. B 81, 1977 (2001).

${ }^{32}$ Z. Fang and K. Terakura, Surf. Sci. 470, L75 (2000).

${ }^{33}$ T. C. Leung, C. T. Chan, and B. N. Harmon, Phys. Rev. B 44, 2923 (1991).

${ }^{34} \mathrm{Ph}$. Dufek, P. Blaha, V. Sliwko, and K. Schwarz, Phys. Rev. B 49, 10170 (1994).

${ }^{35}$ M. D. Towler, N. L. Allan, N. M. Harrison, V. R. Saunders, W. C. Mackrodt, and E. Aprà, Phys. Rev. B 50, 5041 (1994).

${ }^{36}$ A. D. Becke, J. Chem. Phys. 98, 1372 (1993).
${ }^{37}$ E. Heifets, R. I. Eglitis, E. A. Kotomin, J. Maier, and G. Borstel, Surf. Sci. 513, 211 (2002).

${ }^{38}$ J. Muscat, A. Wander, and N. M. Harrison, Chem. Phys. Lett. 342, 397 (2001).

${ }^{39}$ I. P. R. Moreira, F. Illas, and R. L. Martin, Phys. Rev. B 65, $155102(2002)$

${ }^{40}$ R. L. Martin and F. Illas, Phys. Rev. Lett. 79, 1539 (1997).

${ }^{41}$ F. Illas and R. L. Martin, J. Chem. Phys. 108, 2519 (1998).

${ }^{42}$ D. Muñoz, N. M. Harrison, and F. Illas, Phys. Rev. B 69, 085115 (2004).

${ }^{43}$ R. A. Evarestov, S. Piskunov, E. A. Kotomin, and G. Borstel, Phys. Rev. B 67, 064101 (2003).

${ }^{44}$ P. E. Blöchl, Phys. Rev. B 50, 17953 (1994).

${ }^{45}$ G. Kresse and D. Joubert, Phys. Rev. B 59, 1758 (1999).

${ }^{46}$ J. P. Perdew and Y. Wang, Phys. Rev. B 45, 13244 (1992).

${ }^{47}$ H. J. Monkhorst and J. D. Pack, Phys. Rev. B 13, 5188 (1976).

${ }^{48}$ G. Kresse and J. Hafner, VASP Guide (University of Vienna, Austria, 2003).

${ }^{49}$ J. Carrasco, N. Lopez, and F. Illas, J. Chem. Phys. 122, 224705 (2005).

${ }^{50}$ A. D. Becke, J. Chem. Phys. 98, 5648 (1993).

${ }^{51}$ V. R. Saunders, R. Dovesi, C. Roetti, R. Orlando, C. M. ZicovichWilson, N. M. Harrison, K. Doll, B. Civalleri, I. J. Bush, Ph. D'Arco, and M. Llunell, CRYSTAL-03 User Manual (University of Turin, Italy, 2003).

${ }^{52}$ P. J. Hay and W. R. Wadt, J. Chem. Phys. 82, 284 (1985).

${ }^{53}$ S. Piskunov, E. Heifets, R. I. Eglitis, and G. Borstel, Comput. Mater. Sci. 29, 165 (2004).

${ }^{54}$ E. Heifets, R. I. Eglitis, E. A. Kotomin, J. Maier, and G. Borstel, Phys. Rev. B 64, 235417 (2001).

${ }^{55}$ Yu. F. Zhukovskii, E. A. Kotomin, and G. Borstel, Vacuum 74, 235 (2004).

${ }^{56}$ R. F. W. Bader, Atoms in Molecules, A Quantum Theory (Clarendon, Oxford, 1990).

${ }^{57}$ G. Henkelman, A. Arnaldsson, and H. Jónsson, Comput. Mater. Sci. (unpublished).

${ }^{58}$ D. E. Usvyat, R. A. Evarestov, and V. P. Smirnov, Int. J. Quantum Chem. 100, 352 (2004).

${ }^{59}$ V. P. Smirnov and R. A. Evarestov, Phys. Rev. B 72, 075138 (2005).

${ }^{60}$ This has been computed by placing an isolated $\mathrm{O}$ atom in a cubic box and imposing two unpaired electrons, this may be regarded as an approximation to the ${ }^{3} P$ atomic multiplet.

${ }^{61}$ K. Szot, W. Speier, R. Carius, U. Zastrow, and W. Beyer, Phys. Rev. Lett. 88, 075508 (2002).

${ }^{62}$ H. Chevreau, I. P. R. Moreira, B. Silvi, and F. Illas, J. Phys. Chem. A 105, 3570 (2001).

${ }^{63}$ G. Pacchioni, F. Frigoli, D. Ricci, and J. A. Weil, Phys. Rev. B 63, 054102 (2001).

${ }^{64}$ Y. Adachi, S. Kohiki, K. Wagatsuma, and M. Oku, Appl. Surf. Sci. 143, 272 (1999).

${ }^{65}$ J. Carrasco, N. Lopez, and F. Illas (unpublished).

${ }^{66}$ R. I. Eglitis, N. E. Christensen, E. A. Kotomin, A. V. Postnikov, and G. Borstel, Phys. Rev. B 56, 8599 (1997). 